(D) Alice Bouskelá1,2

(D) Jane de Carlos Santana

Capelli ${ }^{1}$

(D) Camilla Medeiros Macedo da Rocha ${ }^{1}$

(D) Flavia Farias Lima ${ }^{1}$

(D) Naiara Sperandio ${ }^{1}$

(D) Vania de Matos Fonseca ${ }^{2}$

1 Universidade Federal do Rio de Janeiro, Curso de Nutrição, Campus UFRJ-Macaé Professor Aloisio Teixeira. Macaé, RJ, Brasil.

2 Instituto Nacional de Saúde da Mulher, da Criança e do Adolescente. Fundação Osvaldo Cruz - Fiocruz. Rio de Janeiro, Brasil

\section{Correspondência}

Alice Bouskelá

alice_bouskela@hotmail.com

Artigo oriundo da tese de doutorado intitulada "Situação alimentar de crianças menores de 2 anos assistidas pela Rede de Atenção Básica de Macaé/RJ", pela Pós-Graduação em Saúde da Criança e da Mulher do Instituto Nacional de Saúde da Mulher, da Criança e do Adolescente (IFF/FIOCRUZ).

\title{
Evolução do aleitamento materno exclusivo nos primeiros 15 anos do século XXI: um estudo no município de Macaé, Rio de Janeiro, Brasil
}

Evolution of exclusive breastfeeding in the first 15 years of the $21^{\text {th }}$ century: a study in the municipality of Macaé, Rio de Janeiro, Brazil

\begin{abstract}
Resumo
O estudo tem como objetivo investigar a taxa de prevalência e a tendência temporal do aleitamento materno exclusivo (AME) em lactentes menores de quatro meses atendidos nas Unidades Básicas de Saúde das Estratégias de Saúde da Família (UBS/ESF) de Macaé, entre 2001 e 2015. Realizou-se estudo de série temporal que avaliou a evolução das taxas de prevalência de AME aos quatro meses, considerando o dado período, com caráter descritivo e uso de dados de base secundária, DATASUS - Banco de Dados do Sistema Único de Saúde via Sistema de Informação da Atenção Básica - SIAB/SUS. A análise de tendência de AME foi realizada por meio do software Jointpoint Regression Program para Windows versão 4.5.0.1. Foram analisados 56.454 registros. Para o total de crianças estudadas, o AME apresentou um aumento estatisticamente significativo de 47,2\% para 69,0\% entre 2001 e 2004. Para o período seguinte, a prática de AME sofreu variações até a prevalência de 73,5\%, em 2015, com valor máximo de 76,8\%, em 2013, mas as mesmas não apresentaram significância estatística nesse intervalo de tempo, com uma taxa anual média de 71,4\% nos últimos
\end{abstract}


dez anos. Uma otimista dinâmica de evolução quanto ao AME se estabeleceu durante mais de uma década no município fluminense, confirmando que o investimento em políticas e em diversas ações de promoção, proteção e apoio podem ter impacto positivo e devem permanecer para o contínuo e necessário aumento da prática de amamentação.

Palavras-chave: Aleitamento Materno. Tendências. Estudos de Séries Temporais. Política de Saúde.

\section{Abstract}

This study aims to investigate the prevalence rate and the temporal trend of exclusive breastfeeding (EBF) in infants under four months of age attended at the Basic Health Units of Family Health Strategies (UBS/ESF) in Macaé, between 2001 and 2015. A time series study was conducted to evaluate the evolution of the prevalence rates of EBF at four months, considering the given period, with descriptive character and use of secondary data, DATASUS - Database of the Unified Health System via Basic Care Information System - SIAB/SUS. The EBF trend analysis was performed using the Jointpoint Regression Program software for Windows version 4.5.0.1. A total of 56,454 records were analyzed. For the total number of children studied, EBF presented a statistically significant increase from 47.2\% to 69.0\% between 2001 and 2004. For the following period, the practice of EBF varied until the prevalence of $73.5 \%$ in 2015 , with a maximum value of $76.8 \%$ in 2013 , but these variations did not show statistical significance in this time interval, with an average annual rate of $71.4 \%$ in the last ten years. An optimistic evolution dynamic regarding EBF has been established for more than a decade in the city of Rio de Janeiro, confirming that investment in policies and in various promotion, protection and support actions can have a positive impact and should remain for the continuous and necessary increase in breastfeeding.

Keywords: Breastfeeding. Trends. Time Series Studies. Health Policy. 


\section{INTRODUÇÃO}

O aleitamento materno (AM) é reconhecido como a mais sábia estratégia natural de vínculo, afeto, proteção e nutrição para a criança, além da mais sensível, econômica e eficaz intervenção para redução da morbimortalidade infantil., ${ }^{1,2}$

As evidências são crescentes ao apontar o leite materno como um valioso alimento funcional a partir de seus inúmeros efeitos fisiológicos e de repercussões positivas ao estado nutricional do lactente, como na sua saúde em curto e longo prazos. ${ }^{3,4}$ Relatórios publicados recentemente pela Organização Mundial da Saúde (OMS) reconhecem tais benefícios, sejam os mesmos de impacto imediato, como pela comprovada redução da incidência e gravidade de infecções intestinais e respiratórias e menor risco de mortalidade nas crianças em aleitamento materno exclusivo (AME), seja pela proteção contra doenças crônicas, como a obesidade. ${ }^{5,6}$

Após uma "epidemia mundial do desmame", decorrente da revolução industrial, esforços nacionais e internacionais se somaram a partir de meados da década de 1970 e no Brasil, em 1981, foi instituído o Programa Nacional de Incentivo ao Aleitamento Materno (PNIAM) com o objetivo de incentivar o AM e reduzir o desmame precoce. Surgiram propostas como a implantação do alojamento conjunto, o início da amamentação imediatamente após o nascimento, a criação de leis sobre creche no local de trabalho e o aumento do tempo da licença maternidade. ${ }^{7,8}$

Enquanto resultado de tais políticas, inquéritos nacionais que tratam das prevalências dos indicadores do AM no país demonstram expansão do AME e seguem com tendência ascendente até 2006, mas com uma estabilização preocupante para os anos subsequentes (2006-2013).9,10

Como um município da Região Norte do estado do Rio de Janeiro, de destaque por sua atividade petrolífera, Macaé tem investido em diversas ações em prol do AM, mas a escassez de estudos sobre a temática interfere na avaliação das políticas e programas locais. Sendo assim, o objetivo deste estudo foi investigar a prevalência e a tendência temporal do AME em lactentes menores de quatro meses, no âmbito da Atenção Básica do município de Macaé, ao longo dos primeiros 15 anos do século XXI.

\section{MÉTODOS}

Foi realizado estudo de série temporal que avaliou a evolução das prevalências de AME, considerando o período de 2001 a 2015. O mesmo apresentou caráter descritivo com o uso de dados de base secundária. O estudo teve como unidade de análise o município de Macaé, 
localizado na região norte do estado do Rio de Janeiro (RJ), conhecido como a "Capital Nacional do Petróleo". ${ }^{11}$

Macaé possui uma área territorial de 1.216,846 $\mathrm{Km}^{2}$ e, pelo último censo demográfico, uma população residente de 206.728 habitantes, sendo a $13^{\mathrm{a}}$ cidade mais populosa do estado do RJ, classificada como de médio porte. A população é predominantemente urbana, com respectivos 202.859 domicílios e 3.869 domicílios rurais, segundo informações do Instituto Brasileiro de Geografia e Estatística (IBGE). ${ }^{12}$ A coleta dos dados ocorreu nos meses de fevereiro a abril de 2017 e foi realizada através do site DATASUS - Banco de Dados do Sistema Único de Saúde via Sistema de Informação da Atenção Básica - SIAB/SUS. ${ }^{13}$

As variáveis captadas em tal banco de dados foram: lactentes menores de quatro meses em AME e lactentes menores de quatro meses assistidos nas Unidades Básicas de Saúde das Estratégias de Saúde da Família (UBS/ESF) do referido local, no período de janeiro de 2001 a dezembro de 2015.

O banco de dados foi construído utilizando-se o software Microsoft Excel for Windows ${ }^{\circledR} 2010$. Na análise descritiva, foram utilizados os dados absolutos de AME, e posteriormente, foram calculadas as prevalências anuais e mensais, conforme demonstrado a seguir.

\section{Prevalências mensais do AME (PM_AME)}

PM_AME = número $\left(\mathrm{n}^{\circ}\right)$ total de lactentes $<$ quatro meses em AME em cada mês x100

$n^{\circ}$ total de lactentes < quatro meses assistidas nas UBS/ESF no respectivo mês

\section{Prevalências anuais do AME (PA_AME)}

PA_AME $=\sum$ prevalências mensais de lactentes $<$ quatro meses em AME x 100

12

A partir dos dados resgatados no DATASUS, a análise de tendência de AME foi realizada por meio do software Jointpoint Regression Program para Windows versão 4.5.0.1, disponibilizado pelo National Cancer Institute - NCl, USA. Essa análise por regressão linear segmentada foi utilizada para identificar os possíveis jointpoints que refletem mudanças significativas na tendência estudada, considerando, como variável regressora, as referidas prevalências e, como independente, o ano. 
O programa foi aplicado para calcular, através da modelagem pelo método Jointpoint, a mudança percentual anual (MPA ou, em inglês, Annual Percentage Change - APC) e avaliar a tendência de cada segmento analisado, estimando os valores estatisticamente significativos considerando um nível de confiança de 95\%. A análise de tendência foi realizada de forma descritiva e de forma analítica, por meio de tabelas e figuras. A descrição considerou "aumento" ou "redução" a partir da significância estatística $(p<0,05)$ da tendência (aclive/declive). E, para os valores de APC não significativos, os mesmos foram considerados "estáveis".

O estudo em questão utilizou dados secundários, de domínio público, disponíveis no DATASUS, sem a possibilidade de identificação de sujeitos, em acordo com a Resolução $n^{\circ}$ 466/2012 do Conselho Nacional de Saúde/Ministério da Saúde. ${ }^{14}$ Não necessitou, assim, de apreciação por parte de um Comitê de Ética em Pesquisa para a respectiva avaliação quanto aos riscos a seres humanos. De qualquer forma, o referido estudo está inserido no projeto de pesquisa "Amamenta e alimenta na Atenção Primária à Saúde do município de Macaé - Rio de Janeiro", aprovado pelo respectivo comitê da Faculdade de Medicina de Campos dos Goytacazes, sob o número CAAE: 30378514.1.0000.5244.

Ademais, o estudo contou com a autorização para a utilização dos dados para análise e divulgação dos resultados, a qual foi concedida pela Coordenadoria da Área Técnica de Alimentação e Nutrição - CATAN/Secretaria Municipal de Saúde de Macaé - SEMUSA Macaé, Rio de Janeiro.

\section{RESULTADOS}

Foram analisados 56.454 registros de lactentes menores de quatro meses assistidos pelas UBS/ESF do município de Macaé-RJ, entre 2001 e 2015. As taxas de prevalências anuais encontradas de AME aos quatro meses estão representadas na tabela 1. Para o total de crianças estudadas, o AME apresentou aumento estatisticamente significativo de 47,2\% para 69,0\% entre 2001 e 2004. Para o período seguinte, a prática de AME sofreu variações até a taxa de prevalência observada de 73,5\% em 2015, com um valor máximo de 76,8\%, em 2013, mas as mesmas não apresentaram significância estatística nesse intervalo de tempo, com uma taxa de prevalência anual média de 71,4\% nos últimos dez anos (figura 1). 
Tabela 1. Taxas de prevalências de aleitamento materno exclusivo (AME) aos quatro meses de idade por ano de estudo. Macaé-RJ, 2001-2015.

\begin{tabular}{ccc}
\hline Ano & Valor observado \% (EP) & Valor modelado \\
\hline 2001 & $47,2(0,9)$ & 47,5 \\
2002 & $52,9(0,9)$ & 54,2 \\
2003 & $64,6(0,8)$ & 61,9 \\
$2004^{b}$ & $69,0(0,7)$ & 70,6 \\
2005 & $69,8(0,7)$ & 70,8 \\
2006 & $72,7(0,7)$ & 71,1 \\
2007 & $72,2(0,7)$ & 71,3 \\
2008 & $70,4(0,7)$ & 71,5 \\
2009 & $72,8(0,8)$ & 71,8 \\
2010 & $72,0(0,8)$ & 72,0 \\
2011 & $68,6(0,7)$ & 72,2 \\
2012 & $71,6(0,7)$ & 72,5 \\
2013 & $76,8(0,7)$ & 72,7 \\
2014 & $70,5(0,8)$ & 72,9 \\
2015 & $73,5(0,7)$ & 73,2 \\
\hline
\end{tabular}

aErro padrão da estimativa; ' ’joinpoint.

Figura 1. Tendência das prevalências de aleitamento materno exclusivo (AME) em lactentes menores de quatro meses de idade, Macaé-RJ, 2001-2015.

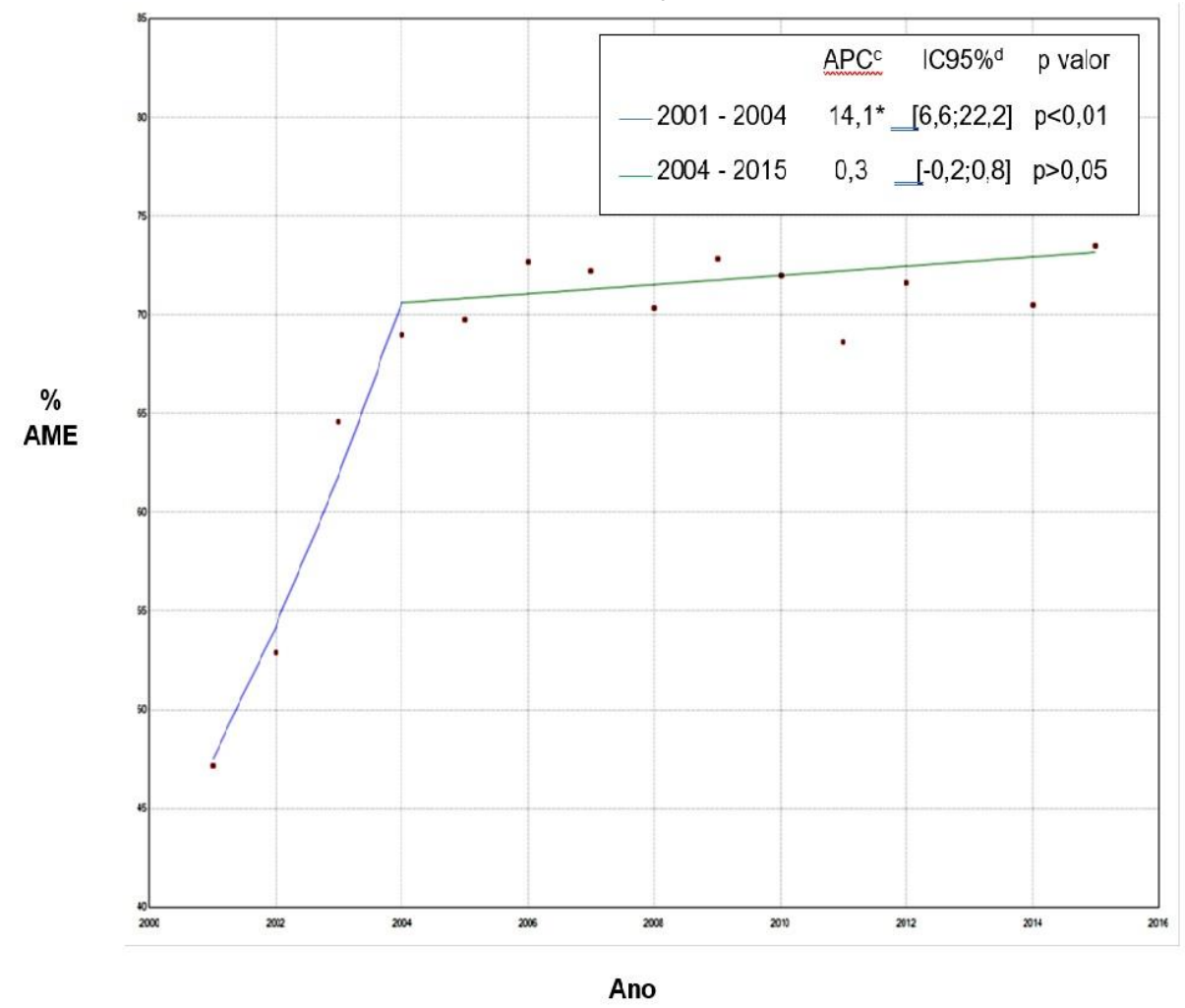

'Annual Percentage Change; 'Intervalo de confiança no nível 95\%; *Dados estatisticamente significativos. 
As taxas de prevalências observadas neste estudo são classificadas como "boas" (50-89\%) de acordo com os indicadores de AM, segundo parâmetros da OMS, publicados em $2008 .^{15}$

\section{DISCUSSÃO}

Este estudo permitiu conhecer a tendência temporal do AME no município de Macaé e analisar tal evolução perante o histórico diferenciado do serviço de saúde local. Entre 2001 e 2004, observou-se uma tendência ascendente com um acréscimo estatisticamente significativo nas taxas de prevalência de AME, alcançando o valor de 69,0\%. No período de 2004-2015, as prevalências de AME se mantiveram estáveis e não apresentaram significância estatística, mas com um resultado positivo de taxa de prevalência anual média de 71,4\%.

Na década de 90, Labbok e colaboradores ${ }^{16}$ avaliaram a tendência do AME, entre 1990 e 2000, em países em desenvolvimento, observando uma elevação de 46,0\% para 53,0\% em crianças menores de quatro meses, e de 34,0\% para 39,0\% em menores de seis meses. Os autores sugerem que as intervenções de apoio à amamentação que marcaram a época do estudo, respaldadas pela implementação da Estratégia Global para a Alimentação de Lactentes e Crianças de Primeira Infância, ${ }^{17}$ foram responsáveis por esse progresso.

Ao avaliar os inquéritos populacionais das décadas anteriores, anos 70 e 80, Venâncio \& Monteiro ${ }^{7}$ retrataram a tendência temporal de duas décadas com relação à amamentação no Brasil e confirmaram o esperado aumento quanto a sua frequência e duração. Entre 1975 e 1989, a duração mediana da amamentação, considerando crianças com um, dois, três, quatro, seis, e 12 meses de idade, foi mais do que duplicada considerando todo o país, passando de dois meses e meio para cinco meses e meio. De forma destacável, em 1975, o percentual de crianças de quatro meses amamentadas foi de 40,0\%, passando para 57,0\% em 1989. Os autores supracitados destacam que o contexto sócio-político-cultural vigente foi apontado como o articulador de tal processo, sendo caracterizado por políticas governamentais próamamentação iniciadas na década de 80, como a criação do PNIAM, responsável por extensa campanha na imprensa.

No âmbito internacional, o Brasil assinou a Declaração de Innocenti, em 1991,18 incorporando a Iniciativa Hospital Amigo da Criança da OMS/Fundo das Nações Unidas para a Infância (UNICEF) às atividades do PNIAM, e promovendo anualmente, no mês de agosto, a Semana Mundial da Amamentação (SMAM). Após este marco, demais eventos importantes ocorreram para a proteção legal do AM, como a aprovação do sistema de alojamento conjunto, em 1993, ${ }^{19}$ e da Norma Brasileira para Comercialização de Alimentos para Lactentes (NBCAL), 
aprovada pelo Conselho Nacional de Saúde em 1988, e regulamentada pela Lei nº 11.265/2006;20 da extensão da licença-maternidade para 120 dias e a criação da licençapaternidade de cinco dias, na Constituição de $1988 .{ }^{21}$

Em momento posterior, demais políticas e programas do governo brasileiro ocorreram a favor do AM, como a Humanização do Pré-natal e Nascimento, ${ }^{22}$ o Método Mãe Canguru, ${ }^{23}$ os Bancos de Leite Humano (BLH), ${ }^{24}$ a Triagem Neonatal, ${ }^{25}$ o Guia Alimentar para crianças menores de dois $\operatorname{anos}^{26,27}$ e a distribuição da Caderneta de Saúde da Criança a todas as crianças nascidas em território brasileiro. ${ }^{28}$

Atualmente, o incentivo ao AM na Atenção Básica acontece por meio da Estratégia Amamenta e Alimenta Brasil (EAAB), do Ministério da Saúde (MS), que é resultante da integração, em 2012, da Rede Amamenta Brasil e a Estratégia Nacional para a Alimentação Complementar Saudável (Enpacs). A EAAB visa reforçar e incentivar a promoção do AM e da alimentação saudável para crianças menores de dois anos no âmbito do Sistema Único de Saúde (SUS), por meio da qualificação dos profissionais atuantes nas UBS. ${ }^{29}$

Entretanto, apesar dos esforços empreendidos em prol do AM, a situação desta prática tão valiosa permanece aquém das metas nacionais e internacionais. ${ }^{30}$ De acordo com a UNICEF e a OMS, ${ }^{31}$ considerando 194 nações, apenas 40,0\% das crianças menores de seis meses de vida são amamentadas exclusivamente; e somente 23 países apresentam taxas de AME acima de $60,0 \%$.

No Brasil, a prevalência de AME em 2008, de acordo com a II Pesquisa de Prevalência de Aleitamento Materno nas Capitais Brasileiras e Distrito Federal, foi de 41,0\%,32 sendo considerado um percentual "razoável" de acordo com a OMS. ${ }^{15}$ Alguns fatores podem vir a contribuir negativamente com essa prevalência, sendo alguns deles: o retorno materno ao trabalho, a falta de apoio por parte da equipe de saúde, a grande veiculação de propagandas de fórmulas, nível socioeconômico, escolaridade e até mesmo aspectos culturais. ${ }^{33}$

Ao atualizar a tendência dos indicadores de AM no Brasil, Boccolini et al. ${ }^{10}$ observaram que as prevalências de AME em lactentes menores de seis meses de vida tiveram tendência ascendente até 2006, ou seja, aumentaram de 4,7\% em 1986 para 37,1\% (em 2006). No entanto, ocorreu relativa estabilização entre 2006 e 2013 (36,6\%), o que deve impor, segundo os autores, uma avaliação e revisão das políticas e programas de promoção, proteção e apoio ao AM.

Na verdade, devido à extensa dimensão territorial brasileira e às diferentes iniciativas locais de promoção do AME, é possível observar oscilações em relação às taxas de AME. Como exemplo, no município de Bauru-SP, a prevalência de AME em menores de seis meses quase 
triplicou no período estudado, passando de 8,5\% em 1999 para 24,2\% em 2006. Para esse desfecho positivo, os autores responsabilizaram as ações organizadas em promoção e apoio ao AM junto às mães do município, assim como investimentos na capacitação de profissionais que atuam na rede pública de Atenção Básica à saúde e em maternidades e na promoção do AM na mídia local mediante organização de eventos. ${ }^{34}$ Queluz et al. ${ }^{35}$ detectaram, no município de Serrana-SP, prevalência de AME em crianças menores de seis meses, no ano de 2009, de 29,8\%, resultado similar ao estudo anterior. Já em Londrina, Paraná-PR, Souza et al. ${ }^{36}$ encontraram uma prevalência de AME de 33,8\% em lactentes menores de seis meses, no ano de 2008, destacando o intenso comprometimento dos profissionais de saúde locais em desenvolver ações para a melhoria dos índices de AM.

Em Pelotas-RS, em 2010, Stephan et al. ${ }^{37}$ verificaram que 33,7\% das mães entrevistadas amamentaram exclusivamente até o sexto mês. Tal estudo foi realizado em uma unidade de Saúde da Família do município e reconheceram que seus resultados não foram diferentes de outros estudos conduzidos no país. Já em Palmas-TO, Borges et al. ${ }^{38}$ constataram que a maioria $(64,0 \%)$ das entrevistadas praticava AME, destacando que esse resultado satisfatório e acima da média nacional foi devido às orientações e ao auxílio dos profissionais do BLH presentes na maternidade pública local, como relatado pelas próprias mães do estudo.

No município de Macaé-RJ, alguns marcos importantes para a Saúde Pública se deram em 2003 e 2004, período de aumento significativo das taxas de AME e que talvez possam justificar tal avanço. No primeiro ano, teve início a comemoração da SMAM no Núcleo de Atenção à Mulher e à Criança (NUAMC), centro especializado de média complexidade do município. Diversas ações de incentivo ao AM passaram a ocorrer ao longo de todo o ano, com o envolvimento irrestrito da respectiva equipe multiprofissional. ${ }^{39} \mathrm{Em} \mathrm{2004}$, foi inaugurado o Hospital Público Municipal Dr. Fernando Pereira da Silva (HPM), referência para a Região Norte Fluminense, tanto para procedimentos de emergência, como a sua própria maternidade. ${ }^{40} \mathrm{O}$ projeto HPMame foi um exemplo de oportunidade para a atualização de todos os profissionais de saúde do município, seja da rede pública ou privada, no tema da amamentação. ${ }^{41}$ No mesmo ano, o MS escolheu Macaé para participar do plano piloto de implantação do novo SIAB, pelo mérito de apresentar uma base de dados muito bem organizada. ${ }^{42}$

Demais ações igualmente colaboram para a manutenção de boas taxas de AME no município, diante do auxílio prestado em centros especializados, como no Centro de Referência do Adolescente (CRA), e da promoção de cursos destinados às gestantes e nutrizes que abordam tal temática. ${ }^{43,44}$ Isso foi possível por meio da Secretaria Municipal de Saúde, a qual promove semelhante incentivo através de outras parcerias, como com o grupo teatral Grutas, 
de atuação em ESF, abordando as dúvidas sobre amamentação com diálogos divertidos. ${ }^{45}$ Outros exemplos de atividades conjuntas seriam com a CATAN e com a Universidade Federal do Rio de Janeiro/Campus UFRJ-Macaé Professor Aloisio Teixeira, iniciadas em 2010, devido à implantação do curso de Nutrição e demais áreas da saúde em agosto de 2009. A partir de 2012, as mesmas se intensificaram e renderam salas de espera, oficinas, palestras e capacitações para o público em geral, para agentes comunitários de saúde e outros profissionais atuantes no município. ${ }^{46,47}$

Por fim, é importante considerar que o sistema de informação em saúde DATASUS/SIAB disponibiliza dados de crianças em AME com idade abaixo de quatro meses acompanhadas pelas UBS/ESF de Macaé, inviabilizando conhecer a realidade do AME em menores de seis meses para o referido município. Ademais, estudos com dados secundários podem apresentar ressalvas quanto à qualidade da informação por subnotificação ou dados incompletos, além de aspectos relativos à falta de padronização na coleta dos dados. Outra limitação refere-se à cobertura da ESF no município, a qual corresponde a aproximadamente 60,0\% da população total residente em Macaé, ao final do período retratado por este estudo. Assim, não é possível extrapolar os resultados encontrados para a totalidade da população de lactentes menores de quatro meses.

\section{CONCLUSÃO}

Os resultados apresentados revelaram uma otimista dinâmica de evolução quanto ao AME durante mais de uma década em um município fluminense, ao início do século XXI. Foi possível demonstrar o quanto essa importante prática pode sofrer alterações positivas ao longo dos anos, sugerindo que o investimento em ações de promoção, proteção e apoio pode, realmente, impactar no aumento de sua prevalência. É fundamental que seja dada continuidade ao incentivo do AM para que os indicadores alcancem patamares elevados.

Não obstante, este estudo retrata um município que reúne um contexto político organizacional diferenciado a favor da amamentação, com um retorno benéfico para a população infantil residente, assim como para toda a comunidade macaense. Estima-se, desta forma, contribuir para a avaliação e reflexão sobre políticas e programas municipais relacionados ao AM.

\section{REFERÊNCIAS}


1. Sociedade Brasileira de Pediatria. Manual de orientação para a alimentação do lactente, do préescolar, do escolar, do adolescente e na escola. 3. ed. Rio de Janeiro: Sociedade Brasileira de Pediatria; 2012.

2. Brasil. Ministério da Saúde. Secretaria de Atenção à Saúde. Departamento de Atenção Básica. Saúde da criança: aleitamento materno e alimentação complementar. 2. ed. Brasília: Ministério da Saúde; 2015.

3. Lönnerdal, B. Breast milk: a truly functional food. Nutrition 2000; 16(7/8):5100-5111.

4. German JB, Dillard CJ, Ward RE. Bioactive components in milk. Curr Opin Clin Nutr Metab Care 2002; 5(6):653-658.

5. Horta BL, Victora CG. Long-term effects of breastfeeding: a systematic review. Geneva: WHO; 2013.

6. Horta BL, Victora CG. Short-term effects of breastfeeding: a systematic review on the benefits of breastfeeding on diarrhoea and pneumonia mortality. Geneva: WHO; 2013.

7. Venâncio SI, Monteiro CA. A tendência da prática da amamentação no Brasil nas décadas de 70 e 80 . Rev Bras Epidemiologia 1998; 1(1):40-49.

8. Brasil. Ministério da Saúde. Programa nacional de incentivo ao aleitamento materno - PNIAM. Brasília: Ministério da Saúde; 1991.

9. Venâncio SI, Saldiva SR, Monteiro CA. Tendência secular da amamentação no Brasil. Rev Saúde Pública 2013; 47(6):1205-8.

10. Boccolini CS, Boccolini PMM, Monteiro FR, Venâncio SI, Giugliani ERJ. Tendência de indicadores do aleitamento materno no Brasil em três décadas. Rev Saúde Pública 2017; 51(108):1-9.

11. Macaé. Prefeitura de Macaé. Capital Nacional do Petróleo. [Acesso em 02 maio 2019]. Disponível em: http://www.macae.rj.gov.br/conteudo/leitura/titulo/capital-nacional-do-petroleo.

12. Instituto Brasileiro de Geografia e Estatística. Censo Demográfico 2010. [Acesso em 08 maio 2019]. Disponível em: https://censo2010.ibge.gov.br/resultados.html.

13. Brasil. Departamento de Informática do SUS (DATASUS). Sistema de Informação da Atenção Básica (SIAB). [Acesso em 29 abr. 2017]. Disponível em: http://www2.datasus.gov.br/SIAB/index.php?area=04.

14. Brasil. Conselho Nacional de Saúde. Resolução n 466 de 12 de dezembro de 2012. Diário Oficial da União. 13 jun. 2013; 1:59.

15. Lauer JA, Betran AP, Barros AJD, Onis M. Deaths and years of life lost due to suboptimal breast-feeding among children in the developing world: a global ecological risk assessment. Publ Health Nutr. 2006; 9(6):673-685. 
16. Labbok MH, Wardlaw T, Blanc A, Clark D, Terreri N. Trends in exclusive breastfeeding: findings from the 1990s. J Hum Lact. 2006; 22:272-276.

17. Organização Mundial de Saúde. Fundo das Nações Unidas para a Infância. Estratégia global para a alimentação de lactentes e crianças de primeira infância. São Paulo: IBFAN Brasil; 2005.

18. World Health Organization. Innocenti declaration: on the protection, promotion and support of breastfeeding. Ecol Food Nutr. 2010; 26(4):271-3.

19. Brasil. Ministério da Saúde. Portaria GM/MS n. 1016. Institui o alojamento conjunto em hospitais e maternidades do SUS. Diário Oficial da União, 01 set. 1993.

20. Brasil. Lei n. 11.265 de 03 de janeiro de 2006. Regulamenta a comercialização de alimentos para lactentes e crianças de primeira infância e também produtos de puericultura correlatos. Diário Oficial da União, 04 jan. 2006.

21. Brasil. Constituição (1988). Constituição da República Federativa do Brasil. Brasília, DF: Senado Federal; 1988.

22. Brasil. Ministério da Saúde. Portaria GM/MS n. 569. Institui o programa de humanização no pré-natal e nascimento - PHPN. Diário Oficial da União, 08 jun. 2000.

23. Brasil. Ministério da Saúde. Portaria GM/MS n. 693. Aprova norma para orientação para implantação do método mãe canguru, destinado a oferecer atenção humanizada ao RN de baixo peso. Diário Oficial da União, 06 jun. 2000.

24. Brasil. Ministério da Saúde. Portaria GM/MS n. 2193. Define estrutura e atuação dos bancos de leite humano. Diário Oficial da União, 15 set. 2006.

25. Brasil. Ministério da Saúde. Portaria GM/MS n. 822. Institui no âmbito Sistema Único de Saúde, o programa nacional de triagem neonatal. Diário Oficial da União, 07 jun. 2001.

26. Brasil. Ministério da Saúde. Secretaria de Política de Saúde. Organização Pan Americana da Saúde. Guia alimentar para crianças menores de dois anos/Secretaria de Políticas de Saúde, Organização Pan Americana da Saúde. Brasília: Ministério da Saúde; 2002.

27. Brasil. Ministério da Saúde. Secretaria de Atenção à Saúde. Departamento de Atenção Básica. Dez passos para uma alimentação saudável: Guia alimentar para crianças menores de dois anos: um guia para o profissional da saúde na atenção básica. Brasília: Ministério da Saúde, 2 ed., 2013.

28. Brasil. Ministério da Saúde. Portaria GM/MS n. 1058. Institui a disponibilização gratuita da caderneta de saúde da criança. Diário Oficial da União, 05 jul. 2005.

29. Brasil. Ministério da Saúde. Portaria n. 1.920, de 5 de setembro de 2013. Institui a Estratégia Nacional para Promoção do Aleitamento Materno e Alimentação Complementar Saudável no Sistema Único de Saúde (SUS) - Estratégia Amamenta e Alimenta Brasil. Diário Oficial da União. 06 set 2013; Seção 1. 
30. Brasil. Ministério da Saúde. Secretaria de Atenção à Saúde. Departamento de Ações Programáticas Estratégicas. Bases para a discussão da Política Nacional de Promoção, Proteção e Apoio ao Aleitamento Materno. Brasília: Ministério da Saúde; 2017.

31. United Nations Children's Fund. World Health Organization. Global Breastfeeding Scorecard. Tracking Progress for Breastfeeding Policies and Programmes. July 2017.

32. Brasil. Ministério da Saúde. Secretaria de Atenção à Saúde. Departamento de Ações Programáticas e Estratégicas. II Pesquisa de Prevalência de Aleitamento Materno nas Capitais Brasileiras e Distrito Federal. Brasília: Ministério da Saúde; 2009.

33. Guimarães AAS, Versiani CC, Pereira, LB, Silva V. Fatores que levam ao abandono do aleitamento materno exclusivo antes dos seis meses de vida: uma revisão de literatura. Revista Digital. Buenos Aires 2013; 18(181).

34. Parizoto GM, Parada CM, Venancio SI, Caravalhaes MA. Trends and Patterns of Exclusive Breastfeeding for Under-6-month-old Children. J Pediatr (Rio J). 2009; 85(3):201-208.

35. Queluz MC, Pereira MJB, Santos CB, Leite AM, Ricco RG. Prevalência e determinantes do aleitamento materno exclusivo no município de Serrana, São Paulo, Brasil. Rev Esc Enferm USP 2012; 46(3):53743.

36. Souza SNDH, Migoto MT, Rossetto EG, Mello DF. Prevalência de aleitamento materno e fatores associados no município de Londrina-PR. Acta Paul Enferm 2012; 25(1):29-35.

37. Stephan AMS, Cavada MN, Vilela CZ. Prevalência de aleitamento materno exclusivo até a idade de seis meses e características maternas associadas, em área de abrangência de unidade de Saúde da Família no Município de Pelotas, Estado do Rio Grande do Sul, Brasil, 2010. Epidemiol Serv Saúde 2012; $21(3): 431-438$

38. Borges NR, Sousa DKS, Pereira RJ, Castro JGD. Caracterização e prevalência do aleitamento materno em uma população atendida na rede pública de saúde de Palmas/TO, Brasil. Rev Bras Pesq Saúde 2016; 18(4):30-36.

39. Macaé. Prefeitura de Macaé. Nuamc Aroeira comemora a Semana do Aleitamento Materno. [Acesso em 04 jun. 2019]. Disponível em: http://www.macae.rj.gov.br/noticias/leitura/noticia/nuamc-aroeiracomemora-a-semana-do-aleitamento-materno.

40. Macaé. Prefeitura de Macaé. Maternidade do HPM ganha novos equipamentos. [Acesso em 04 jun. 2019]. Disponível em: http://www.macae.rj.gov.br/saude/leitura/noticia/maternidade-do-hpm-ganhanovos-equipamentos.

41. Macaé. Prefeitura de Macaé. Curso do HPM orienta profissionais sobre amamentação. [Acesso em 04 jun. 2019]. Disponível em: http://www.macae.rj.gov.br/noticias/leitura/noticia/curso-do-hpmorienta-profissionais-sobre-amamentacao. 
42. Macaé. Prefeitura de Macaé. Ministério da Saúde elogia Programa de Saúde da Família de Macaé. [Acesso em 04 jun. 2019]. Disponível em: http://www.macae.rj.gov.br/noticias/leitura/noticia/ministerio-da-saude-elogia-programa-de-saudeda-familia-de-macae.

43. Macaé. Prefeitura de Macaé. Saúde intensifica ações de promoção ao aleitamento materno. [Acesso em 05 jun. 2019]. Disponível em: http://www.macae.rj.gov.br/noticias/leitura/noticia/saudeintensifica-acoes-de-promocao-ao-aleitamento-materno

44. Macaé. Prefeitura de Macaé. Continuam abertas inscrições para curso de gestantes e lactantes. [Acesso em 05 jun. 2019]. Disponível em: http://www.macae.rj.gov.br/noticias/leitura/noticia/continuam-abertas-inscricoes-para-curso-degestantes-e-lactantes

45. Macaé. Prefeitura de Macaé. Peça Dona Mama destaca amamentação na Semana do Aleitamento Materno. [Acesso em 05 jun. 2019]. Disponível em: http://www.macae.rj.gov.br/noticias/leitura/noticia/peca-dona-mama-destaca-amamentacao-nasemana-do-aleitamento-materno.

46. Lourenço AE, Cordeiro AA, Capelli JCS, Oliveira RBA, Pontes PV, Almeida MFL, Barros LB. Programa de Educação pelo Trabalho para a Saúde (PET-Saúde) e a formação do nutricionista num campus de interiorização. Demetra 2017; 12(1):41-58.

47. Capelli JCS, Rocha CMM, Braga FAMN, Lima FF, Rodrigues VS, Almeida MFL, Sperandio N, Monteiro LS, Viana MR, Latorre CG, Bouskela A. Ações de promoção da alimentação saudável no primeiro ano de vida em Macaé. Revista da Associação Brasileira de Nutrição - RASBRAN. 2018; 9(2):9-16.

\section{Colaboradores}

Bouskelá A, Capelli JCS e Rocha CMM participaram de todas as etapas: desde a idealização do desenho do estudo, participação na coleta, análise e interpretação dos dados, participação na redação do estudo e na revisão final do manuscrito. Lima FF, Sperandio N participaram da interpretação dos dados, da redação e revisão do manuscrito. Fonseca VM participou da revisão final do manuscrito.

Conflitos de interesses: Os autores declaram não haver conflito de interesses.

Recebido: 26 de junho de 2019

Revisado: 15 de setembro de 2019

Aceito: 19 de setembro de 2019 